\title{
Thoracoscopy Assisted Minimally Surgery (NUSS procedure) for Pectus Excavatum vs. Novel Modified NUSS procedure - A Single-Center Retrospective Study
}

\author{
Lifei Meng, Mingsong Wang \\ Department of Cardiothoracic Surgery, Xinhua Hospital, Shanghai Jiaotong University. Shanghai, China
}

\section{ABSTRACT}

Objective: The purpose of this article is to evaluate the efficacy of thoracoscopy assisted minimally surgery (NUSS procedure) for pectus excavatum and novel modified NUSS procedure by comparing the data of patients of pectus excavatum who had undergone novel modified NUSS Procedure or NUSS procedure.

Methods: A retrospective study was performed, involving 132 cases of patients with pectus excavatum collected from Shanghai Jiaotong University School of Medicine Xinhua Hospital cardio-thoracic surgery between Jan. 2009 and Jan. 2012. The 132 patients were strictly divided into two groups: Group A included 76 cases that underwent a novel modified NUSS procedure; Group B included 56 cases that underwent NUSS procedure. Compared data included gender, age, operative time, blood loss, postoperative hospital staying and clinical variables and demographic were compared with univariable analysis.

Results: No statistically significant correlations were observed in the two groups, such as age $(12.67 \pm 4.793$ years vs. $12.20 \pm 6.423$ years), sex, and other clinical data $(\mathrm{P}>0.05)$. In Group A, postoperative hospitalization time $(3.95 \pm 0.487$ days vs. $6.07 \pm 1.412$ days), operation time $(46.28 \pm 12.218 \mathrm{~min}-$ utes vs. $72.23 \pm 24.270$ minutes), and blood loss $(7.37 \pm 4.863$ $\mathrm{ml}$ vs. $16.93 \pm 14.002 \mathrm{ml}$ ) were significantly better than those in Group B $(\mathrm{P}<0.05)$, shortening hospitalization time, reducing costs, meaning statistically significant differences. There was no recurrence in Group A and Group B after operation.

Conclusion: The study demonstrated that novel modified NUSS procedure is less invasive, having rapid recovery, shortening the time of hospital stay, and reducing hospital cost. Thus, it is safe, worth promoting, and is widely being used.

Received March 7, 2018; accepted fuly 12, 2018.

Correspondence: Mingsong Wang, Department of Cardiothoracic Surgery, Xinhua Hospital, Shanghai fiaotong University. Shanghai 200092, China; (e-mail: wangmingsong@xinhuamed.com.cn).

\section{INTRODUCTION}

Pectus excavatum is one of the most common congenital thoracic deformity diseases accounting for the incidence of 0.1 percent [Zuidema 2018]. Surgery is the only effective way to cure the disease, in which NUSS procedure (thoracoscopy assisted minimally surgery for pectus excavatum) is thought to be the most popular minimally invasive technique [Tedde 2012; Pawlak 2013; Pilegaard 2016]. However, installation or removal of the bar of the traditional NUSS procedure sometimes is difficult and traumatic [Ohno 2009].

Since 2010, the novel modified NUSS procedure was independently introduced by ourselves to do the correction of pectus excavatum on the basis of traditional NUSS procedure. It was reported that the new steel bar could be easily installed or removed [ $\mathrm{Li} 2015]$, shortening operation time and reducing trauma, thus it also easy to be accepted by patients and their families [Lobe 2006]. But the efficacy of comparing data of novel modified NUSS procedure and traditional NUSS procedure is rarely reported, so we retrospectively summarized surgical efficacy data here. It is important to explore treatment strategies and choose the right operation mode.

\section{METHODS}

A retrospective study was performed, involving 132 cases of patients with pectus excavatum collected from Shanghai Jiaotong University School of Medicine Xinhua Hospital cardio-thoracic surgery between Jan. 2009 and Jan. 2012. The 132 patients were strictly divided into two groups: Group A included 76 cases that underwent a novel modified NUSS procedure; Group B included 56 cases that underwent NUSS procedure. Compared data included gender, age, operative time, bleeding volume, days of postoperative hospital stay, and recurrence. The study was approved by hospital ethics committee, and patients signed a consent form.

The main inclusion criteria included (1) preoperative CT index $>3.2$; (2) the thoracic deformity developed gradually; and (3) preoperative imaging and heart super suggested oppression of cardiopulmonary.

Patients were excluded if they (1) were diagnosed with a serious lung infection by abnormal blood biochemical; (2) had severe cardiopulmonary dysfunction that could not 
$1 \mathrm{~A}$

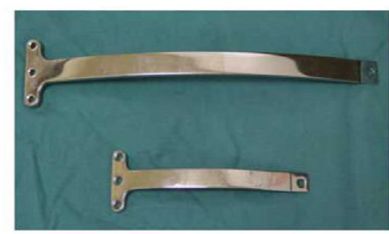

$1 \mathrm{~B}$

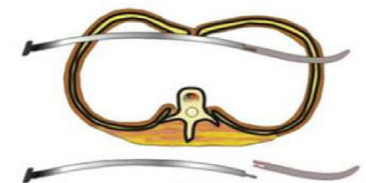

(1)
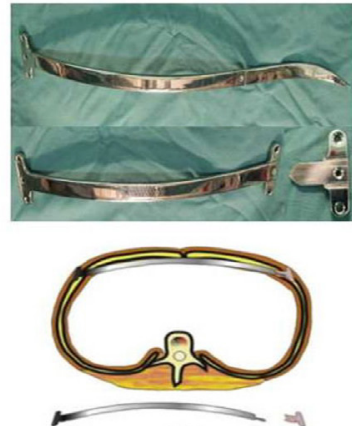

(2)

(1) The modified Nuss bar was inserted into the right thoracic cavity and woven behind the sternum anterior to the pericardium through the bilateral pleural cavity. (2) The shape of the chest wall and the sternum sag immediately changed after the bar was pushed in and pulled out through the tunnel after the introducer without turning it over. Last, the introducer was removed and stabilizer was placed to fix the bar.

tolerate surgery; and (3) had an impaired general health status and/or lost follow up.

Operative techniques - Modified NUSS procedure: Under general anesthesia, lateral incisions of $2 \mathrm{~cm}$ to 3 $\mathrm{cm}$ were made on both sides of the chest, and the incisions were deep and reached the ribs. Electrocautery was used to dissect the major muscles of the sternum to separate the rib space from the highest point of the thorax, which was at the same level as the lowest point of the sternum. Then, a hole was punched into the right axillary midline of the seventh rib to insert the thoracoscopy as a guide. The new steel bar was placed in the rib gap from the right space. Then, the bar was inserted into the right chest between the ribs at the highest point of the sternum, across the mediastinum of the lowest breastbone, through the chest wall between the peak from the left rib (high and low points of the sternum at the same level), and out of the left rib gap and left incision. The shape of the chest wall and the sternum sag immediately changed. After uninstalling the guide device, the chest plate was set and connected to a fixed piece using screws. After draining the right thoracic cavity, the incisions were closed using the interrupted suture technique (Figure 1).

Operative techniques - Traditional NUSS procedure: Under general anesthesia, a hole was punched into the chest at the right axillary midline of the seventh rib to insert the thoracoscopy to view the lower sternum sag. Two long and longitudinal incisions of $3 \mathrm{~cm}$ were made on both sides of the chest, slightly above the xiphoid process. The incisions were deep and reached the ribs. Electrocautery was used to dissect the major muscles of the sternum gap along the direction of the ribs to the xiphoid process to separate the thoracic peak point and the low point of the sternum at the same level. The device, including NUSS bar, was inserted starting from the right along the clearance to the highest point between the ribs into the right chest, from behind the xiphoid process (low) through the mediastinum, from the left side of the peak through the chest wall along the left

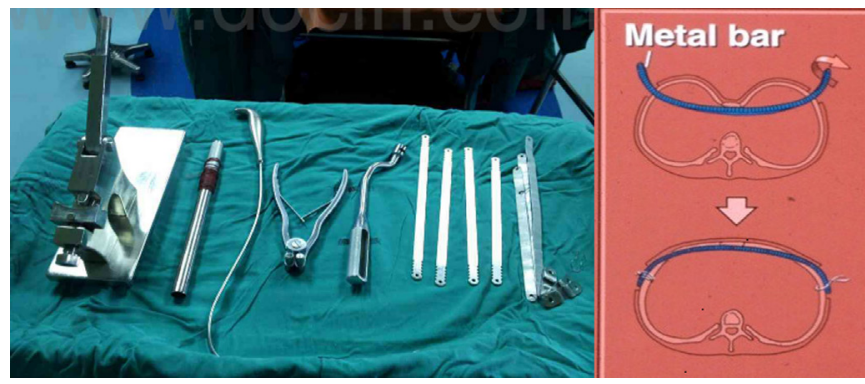

Figure 2. The traditional NUSS bar was inserted starting from the right along the clearance to the highest point between the ribs into the right chest, from behind the xiphoid process (low) through the mediastinum, from the left side of the peak through the chest wall along the left side of the gap between ribs and through the incision on the left side. The shape of the chest wall and the sternum sag immediately changed. After uninstalling the guide device, the chest plate was set and connected to a fixed piece using screws.2. Survival rates. The 1-year and 4-year survival rates were both $95.7 \%$.

side of the gap between ribs and through the incision on the left side. The shape of the chest wall and the sternum sag immediately changed. After uninstalling the guide device, the chest plate was set and connected to a fixed piece using screws. The operative field was checked to ensure no bleeding and no leakage. The chest was rinsed. After draining the right thoracic cavity, the incisions were closed using the interrupted suture technique (Figure 2).

Statistical analysis: We evaluated the operative techniques' effect about the following clinical outcomes: operation time which was defined as time between the beginning of first port incision and the end of completion of wound sutured; blood loss which was defined by calling the weight of the cotton ball to absorb blood and amount of blood in the attractor; postoperative hospitalization time which was defined as time between the next day of the operation and the end day of the hospital; perioperative mortality was due to related or unrelated causes to the disease or procedure within two months of surgery.

Statistics of the collected data are mainly represented by the numbers and percentages, standard deviations (SD), or the median and interquartile range (IQR). Continuous variables were depicted based on IQR and the median or SD and mean. Wilcoxon's rank-sum test was used for ranked variables and Two-sample Student's t-test was used to compare the means of population between two different continuous variables. Chi-squared or Fisher's exact test was also used to evaluate categorical variables, which have statistically significant differences. All hypothesis testing were two-sided, with a $\mathrm{P}$-value of $<0.05$ considered statistically significant differences.

\section{RESULTS}

\section{Demographics and Clinical Characteristics}

From Jan. 2009 to Jan. 2012, 132 patients with the surgery of pectus excavatum at our institution were eligible for the current analysis. Demographics, clinical laboratory, and anesthetic characteristics are in Table 1. 
Table 1. Baseline characteristics of patients and Univariable Analysis of Surgery Data

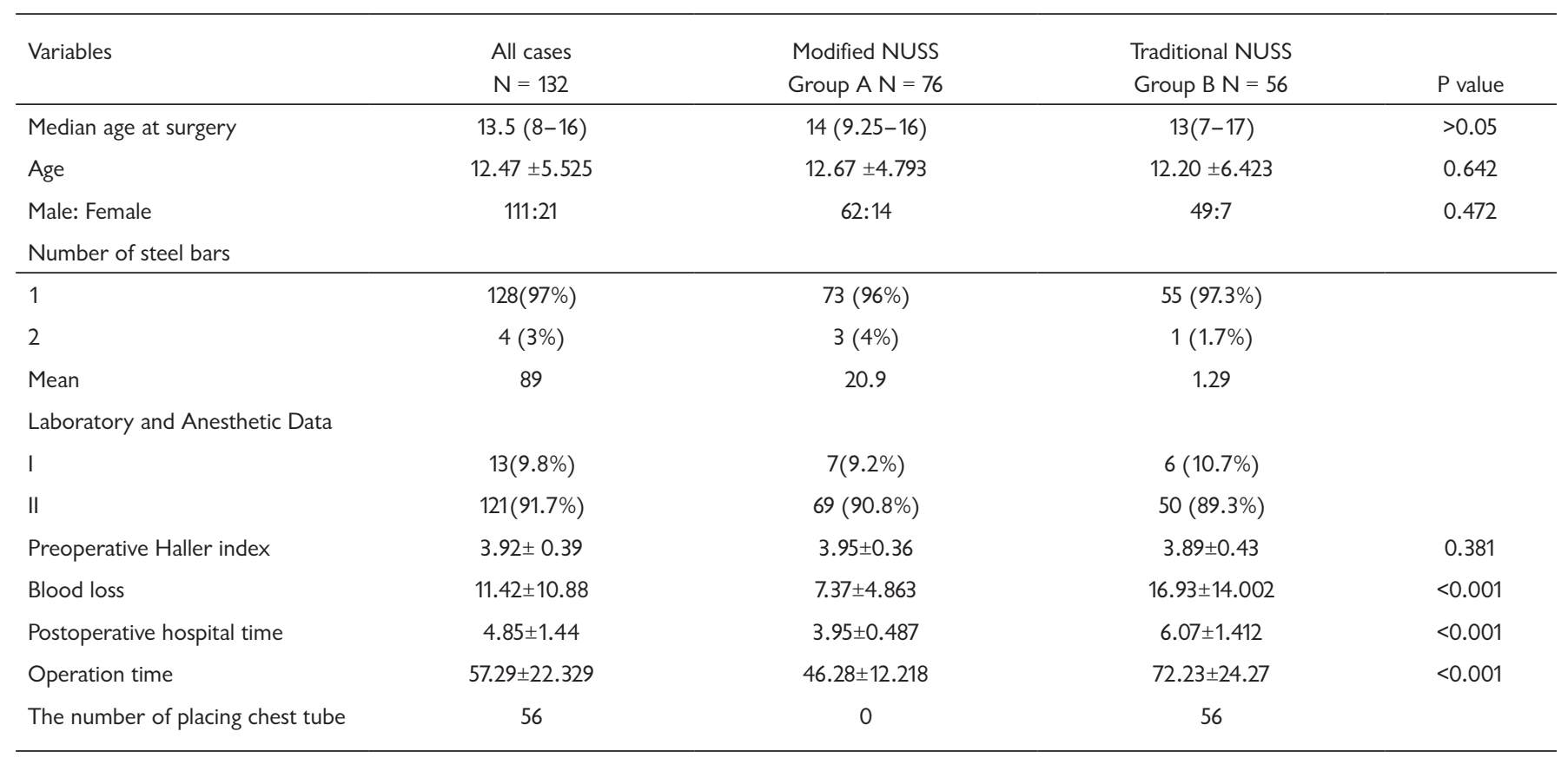

There were 111 male and 21 female patients. Patients ranged from 3 years to 35 years (mean, $12.47 \pm 5.525$ years; median,13.5 years). Of these patients, statistically negative correlations were found in age and surgery modes. No statistically significant correlations were observed in the two groups between sex, age, American Society of Anesthesiologists (ASA) grade and computed tomographic index (CI) (Haller index) with $\mathrm{P}$-value $>0.05$. All pectus excavatum patients were successful to undergo modified NUSS procedure or traditional NUSS procedure. One hundred twenty-eight patients (97 percent) had one steel bar, and four patients had two bars. Among the patients who underwent two bars' surgery, three patients were in the modified NUSS group and one patient was in the traditional NUSS procedure group. When analyzed in terms of blood loss, operation time and postoperative hospital time, modified NUSS group in postoperative hospitalization time $(3.95 \pm 0.487$ days vs. $6.07 \pm 1.412$ days), operation time $(46.28 \pm 12.218$ minutes vs. $72.23 \pm 24.270$ minutes), blood loss $(7.37 \pm 4.863 \mathrm{ml}$ vs. $16.93 \pm 14.002 \mathrm{ml})$ were significantly better than those in the traditional NUSS group $(\mathrm{P}<0.05)$. All patients in the traditional NUSS group were placed with a chest drainage tube after operation. Patients in the modified NUSS group did not need to be placed with the tube.

Postoperative complications of excavatum complications: Table 2 shows various pectus excavatum complications noted during the investigation. Early surgical complications in this study refer to the complications, which occurred within one month of the correcting surgery of pectus excavatum. A total of six patients (4.5 percent) experienced early surgical complications. In Group A, one patient experienced postoperative pneumothorax, which was resolved without chest tube placement. By comparison, in Group B, one patient also experienced pneumothorax, and recovered after chest drainage tube removal. But in both Groups A and B, there was one patient with wound seroma in the traditional NUSS group. The wound infection and wound seroma were treated by debridement surgery and daily changing of dressings. In our series, no case of bar displacement and recurrence was reported.

\section{DISCUSSION}

Funnel chest is one of the most common congenital thoracic deformity diseases; it usually shows the sternum sag

Table 2. Postoperative complications of pectus excavatum

\begin{tabular}{lcc}
\hline Complication & $\begin{array}{c}\text { Modified NUSS } \\
\text { Groups } \\
\text { (Group A) N }=76\end{array}$ & $\begin{array}{c}\text { Traditional NUSS } \\
\text { Group } \\
\text { (Group B) N }=56\end{array}$ \\
\hline Pneumothorax & 1 & 1 \\
Wound infection & 1 & 1 \\
Pleural effusion with a & 1 & 0 \\
little atelectasis & 0 & 1 \\
Wound seroma & 0 & 0 \\
Perioperative mortality & 0 & 0 \\
Recurrence & 0 & 0 \\
Bar obvious displacement & & \\
\hline
\end{tabular}


of the anterior chest wall as the center, shaped as a funnel. Patients with this can experience a corresponding chest tightness, shortness of breath, decreased activity tolerance, and other symptoms. Surgery is the only way to cure the disease [Jacobsen 2010; Weber 2006].

At present, the traditional NUSS procedure is thought to be the most popular minimally invasive surgery of funnel chest [Tedde 2012; Gupta 2012]. But the operation of making the steel plate into the human body is complicated. It requires several hours and needs aggressive separation of muscles and cartilage. When the bend steel plate bypassed the mediastinum lesions, it may increase the incidence of mediastinal hemorrhage and pneumothorax [Wisman 2013; Baek 2017]. And, the steel gravity point is on the intercostal muscles, which can aggravate pain and not be strong enough support these muscles. Furthermore, the bend steel plate will limit growth and development of the chest wall. When the steel plate turns over, huge soft tissue injury could happen at the intercostal muscles [Hakala 2002; Kyutoku 2002], which would aggravate injury. Placing a chest tube after surgery will aggravate the pain, and the pain made it difficult for patients to cough out the sputum. Thereby increasing the risk of postoperative pulmonary complications, and the placement of chest tube led to a decline in patients with postoperative mobility [Ishigaki 2015], which resulted in deep vein thrombosis [Bjerregaard 2014; Olgac 2014]. The traditional NUSS procedure not only increased the number of patients with postoperative discomfort, but also extended the length of hospital stay and increased the economic burden to patients. In addition, the operation of removing NUSS bar is complex and caused large trauma.

Since 2010 and compared with the traditional NUSS procedure, the novel modified NUSS procedure was independently introduced by ourselves to do the correction of pectus excavatum on the basis of NUSS procedure.

The aim was to further optimize on NUSS procedure, reflected in the following points. First, the length of surgical incision in the skin shortens a third, and the tunnel of separating muscle and subcutaneous also decreases in size by half. Second, the installation of the steel plate needs not to turn over, thereby reducing operating difficulty and trauma. Third, the steel plate is easy to install and fixed, the other end socket plate can adjust the length of steel plate, and the elevating degree of steel plate can make for a more accurate fixed position. Fourth, there is no point of view to plates pass mediastinal tissue, which causes small damage, and will not cause mediastinal hemorrhage and complications such as the contralateral pneumothorax. Fifth, steel plates are supported by frame and intercostals muscles, which will not cause intercostal muscle tearing and displacement of the steel plate, and the strong point in the chest wall does not limit the development of the ribs and chest wall. Finally, the correcting new steel bar and fixed piece can be removed along a previous tunnel. Therefore, it is easy to complete the operation. Compared with previous NUSS procedures, it isn't necessary to put in the chest tube, which is helpful for patients to shorten the postoperative hospitalization stay and reduce economic burden to patients.

In the present study, we retrospectively analyzed operation data for nearly three years, collecting 132 cases of patients with surgery of pectus excavatum. Modified group in operation time, blood loss, postoperative hospital stay was better than the traditional NUSS group, with a P-value $<0.05$. The meaning statistically significant, which further proved the novel modified NUSS procedure was better than that of the NUSS procedure. But the bleeding and operating time depends on the proficiency of surgeons, only postoperative hospital stay and no postoperative chest drainage tube could reflect the degree of minimally invasive surgery and effect in modified NUSS group. Reported complications were involved in pneumothorax, wound infection, pleural effusion, pericarditis, pericardial effusion, recurrence, bar obvious displacement and so on [Bean 2015; Lee 2011]. Previous studies have reported the complications were in up to 9 percent [Watanabe 2004]. In our study, incidence of surgical complications was relatively low, accounting for 4.5 percent. Maybe it was mainly due to the insufficient sample size. With the sample size becoming larger, we will do further study.

\section{CONCLUSION}

The novel modified NUSS procedure can reduce postoperative pain, reduce the length of hospital stay, and reduce the economic burden to patients. Therefore, it is safe, effective, and worthy of wide use.

\section{REFERENCES}

Baek JH, Lee YU, Jung TE, et al. 2017. Late cardiac tamponade following Nuss procedure for pectus excavatum. J Thorac Dis 9 (5), E424.

Bean JF, Wax D, et al.2015. Arteriovenous Fistula: A Rare Complication After Nuss Procedure for Pectus Excavatum. Ann Thorac Surg 100 (4), 1463.

Bjerregaard LS, Jensen K, Petersen RH, et al. 2014. Early chest tube removal after video-assisted thoracic surgery lobectomy with serous fluid production up to $500 \mathrm{ml} /$ day. Eur J Cardio-Thora Surg 45 (2), 241-6.

Gupta PK, Ramanan B, Lynch TG, et al. 2012. Development and validation of a risk calculator for prediction of mortality after infrainguinal bypass surgery. J Vasc Surg 56 (2), 372-379.

Hakala T, Pitkänen O, Hippeläinen M. 2002. Feasibility of predicting the risk of atrial fibrillation after coronary artery bypass surgery with logistic regression model. Scand J Surg 91 (4), 339.

Ishigaki D, Arimoto T, Iwayama T, et al. 2015. Prevention of immediate recurrence of atrial fibrillation with low-dose landiolol after radiofrequency catheter ablation. J Arrhythm 31 (5), 279.

Jacobsen EB, Thastum M, Jeppesen JH, et al. 2010. Health-related quality of life in children and adolescents undergoing surgery for pectus excavatum. Eur J Pediatr Surg 20 (2), 85-91.

Kyutoku S, Yasu KM.2002. Surgical treatment of pectus excavatum: Sternal elevation and the Nuss procedure 45 (6), 541-547.

Lee SH, Ryu SM, Cho SJ. 2011. Thoracic Outlet Syndrome After the Nuss Procedure for the Correction of Extreme Pectus Excavatum. Ann Thorac Surg 91 (6), 1975-7.

Li G, Jiang Z, Xiao H, et al. 2015. A novel modified Nuss procedure for pectus excavatum: a new steel bar. Ann Thorac Surg 99 (5), 1788. 
Lobe T. 2006. Perioperative hypnosis reduces hospitalization in patients undergoing the Nuss procedure for pectus excavatum. J Laparoendosc Adva 16 (6), 639-42.

Ohno K, Nakamura T, Azuma T, et al. 2009. Modification of the Nuss procedure for pectus excavatum to prevent cardiac perforation. J Pediatr Surg 44 (12), 2426-2430.

Olgac G, Cosgun T, Vayvada M, et al. 2014. Low protein content of drainage fluid is a good predictor for earlier chest tube removal after lobectomy†. Interact Cardiov Th 19 (4).

Pawlak K, Zieliński P, Gabryel P, et al. 2013. Early and late results of the Nuss operation for pectus excavatum - A single institution experience. Kardiochir Torakochi 10 (4), 364-368.

Pilegaard HK.2016. Minimal Invasive Repair of Pectus Excavatum. Springer International Publishing.
Tedde ML, Campos J R,Wihlm JM, Jatene FB.2012. The Nuss procedure made safer: an effective and simple sternal elevation manoeuvre. Eur J Cardio-Thora 42 (5), 890-1.

Watanabe T, Obama T, Ohsawa H. 2004. The use of a lateral stabilizer increases the incidence of wound trouble following the Nuss procedure. Ann Thorac Surg 77 (1), 296-300.

Weber PG, Huemmer HP, Reingruber B. 2006. Forces to be overcome in correction of pectus excavatum. J Thora Cardiov Surg 132 (6), 1369-1373.

Wisman PP, Hattum ES, Graaf YV, et al. 2013. Derivation and validation of a risk chart for future ischaemic events and mortality following peripheral bypass surgery. Eur Heart J 34 (suppl 1), P5167-P5167.

Zuidema WP, Oosterhuis JW, Zijp GW et al. 2018.Early Consequences of Pectus Excavatum Surgery on Self-Esteem and General Quality of Life. World J Surg 0364-2313. 